\title{
nature
}

\section{Time to grasp the international perspective on GM crops}

Among the clamour of voices from around the globe for and against GM crops, many scientific academies have been quiet. Their views are urgently needed in determining the long-term research agenda.

$\mathrm{N}$ ext month sees an event that should have happened years ago: scientists representing national academies from developed and developing countries are to meet to discuss the way forward for global agricultural biotechnology. The agenda includes discussions on the technology's promises, its potential risks and its regulation. Participants will consider adopting a common position on those issues and the scientific uncertainties in genetically modified (GM) crops. They may even agree to coordinate an international study, for example into the environmental implications of genetic modification in agriculture.

The importance of this meeting — and of such a study — can hardly be overstated. It is commonly argued that GM crops are integral to the future of world food production and have a major role to play in feeding the hungry. But the institutional voices carrying this message — such as Britain's Royal Society, and more recently the Nuffield Council on Bioethics — have come largely from the developed world.

Some influential non-government organizations - such as the Third World Network and the Research Foundation for Science, Technology and Natural Resource Policy in India - have disputed the vision that GM crops are needed to alleviate hunger and malnutrition. Those organizations, like their counterparts in the developed world, claim that hunger is caused because the poor are unable to get access to food, and not necessarily because of a shortage of production.

While many prominent individual scientists have made their views clear, the perspectives of the developing world's leading science academies have been largely absent. Next month's meeting should help to correct that anomaly.

But there is arguably a second, and equally important, reason why next month's meeting could not be more timely: the United States is threatening Europe with — another - trade war unless states of the European Union relax regulations on the sale of GM crops, which are stricter than those on the opposite side of the Atlantic.

European countries cite broad environmental concerns, and last week heads of state of Europe's G8 countries tried to persuade the United States to set up an international committee of scientists to validate new foods (see page 717). The US government, on the other hand, believes Europe's environmental concerns are little more than a smokescreen for old-style protectionism.

Neither side is able to draw on a truly global scientific assessment of potential environmental risks from GM crops because, as yet, none exists. Indeed, the absence of a credible international scientific assessment on the issue contributed to the collapse of biosafety talks earlier this year. Europe, Africa and the United States disagreed on even the most fundamental question: whether GM organisms contribute to global biodiversity loss.

Countries will resume discussions on the biosafety protocol in May 2000. But without such a study, it is difficult to see the meeting achieving a positive result. An international scientific review of the environmental impacts of GM crops is urgently needed, and science academy representatives meeting next month have the opportunity to take the initiative. They should set the ball rolling without delay.

\section{Confrontation out, pragmatism in}

\section{A year-long 'stand-off' between French researchers and the science ministry signals the need for change.}

ust three weeks ago, the French science ministry created a special FF40 million (US\$6.3 million) fund for grants to young researchers with original ideas in any field. It has been taken aback by the scale of the demand — some 1,000 proposals have already been received. This small injection of oxygen has clearly set alight many young minds who are otherwise too often stifled by a patriarchal French laboratory system where the grip of powerful laboratory directors on the strings of the purse and the ideas suffocates innovation.

There is also a message here for a country where everyone is growing tired of a repetitive cycle where the government introduces sweeping reforms, researchers take to the street to oppose them, and deadlock sets in until the next government arrives and the ritual begins over again. That message is that pragmatic and well-targeted reforms may be a more effective way forward than grandiose rearrangements of the research administration.

A similar conclusion seems to be emerging from a national consultation, ordered by Lionel Jospin, the prime minister, in a bid to find a way out of the current deadlock over proposed reforms by Claude Allègre, the science minister. Organized by members of par- liament Pierre Cohen and Jean-Yves Le Déaut — who are also working scientists - the consultation will culminate this weekend with a national colloquium in Paris. The take-home message is likely to be that modernizations of practices, each tuned to particular situations at the grassroots, are needed more than high-profile reforms of, for example, whole funding agencies.

Ironically, the problems acknowledged as priorities by researchers are virtually identical to those that most trouble Allègre's ministry. The lack of independence of young scientists is one. The bloated system of evaluation is another. And then there is the long-recognized rigidity of a system where some scientists enjoy full-time posts in the research agencies while their colleagues in the universities - often young scientists in their prime - struggle to fit research in alongside excessive teaching loads.

Valuable months have been lost as a result of a sterile confrontation between researchers and the ministry. For the sake of science and of government credibility, this weekend's meeting must herald a more productive period where the ministry and the research community can identify critical areas where tangible progress can be achieved. 